\title{
Helix Inspired 28 GHz Broadband Antenna with End-Fire Radiation Pattern
}

\author{
Hijab Zahra', Wahaj Abbas Awan', Niamat Hussain ${ }^{3, *}$, Syed Muzahir Abbas ${ }^{1,4}$ \\ and Subhas Mukhopadhyay ${ }^{1}$
}

\footnotetext{
${ }^{1}$ School of Engineering, Faculty of Science and Engineering, Macquarie University, Sydney, NSW, 2109, Australia ${ }^{2}$ Department of Integrated IT Engineering, Seoul National University of Science and Technology, Seoul, 01811, Korea ${ }^{3}$ Department of Information and Communication Engineering, Chungbuk National University, Chungbuk, 28644, Korea

${ }^{4}$ BENELEC, Botany, Sydney, NSW, 2019, Australia ${ }^{*}$ Corresponding Author: Niamat Hussain. Email: hussain@chungbuk.edu.kr Received: 15 April 2021; Accepted: 24 May 2021
}

\begin{abstract}
This paper presents the design and characterization of a via free planar single turn helix for $28 \mathrm{GHz}$ broadband applications. The proposed antenna is designed using ROGERS RO4003 material, having a simple structure and end-fire radiation pattern. The antenna comprises of a compact dimension of $1.36 \lambda_{0} \times 0.9 \lambda_{0}$ with a thickness of $0.0189 \lambda_{0}$ (where $\lambda_{0}$ is the free-space wavelength at the central frequency of $28 \mathrm{GHz}$ ). Parametric study has been carried out to investigate the impact of key design parameters and to achieve an optimum design. Results show a good agreement between the simulated and measured results. A single turn helical inspired antenna covers $-10 \mathrm{~dB}$ impedance bandwidth of $26.25-30.14 \mathrm{GHz}$ having a peak gain of $5.83 \mathrm{~dB}$ and radiation efficiency up to $85 \%$. Moreover, linear array configurations with 2 and 4 elements have been analyzed for applications with higher gain and space constraints. Presented array configurations are suitable for applications having space constraints in one dimension. Results show that peak gain up to $8.2 \mathrm{~dB}$ and $11.1 \mathrm{~dB}$ can be achieved with 2 and 4 elements, respectively. Due to its simple planar and via free structure, this antenna is suitable for $5 \mathrm{G}$ communications and for sensing, imaging, IoT and tracking applications at $28 \mathrm{GHz}$ band spectrum.
\end{abstract}

Keywords: Compact antenna; millimeter wave antenna; $28 \mathrm{GHz} ; 5 \mathrm{G}$ antenna

\section{Introduction}

The fifth generation (5G) of mobile communications promised higher Gbps data rates with very low latency to support the ever-increasing number of users. The higher data rates are directly associated to bandwidth, therefore radio frequency (RF) front ends operating at millimeter-wave part of the spectrum have drawn significant attention of the researchers [1]. It also provides better communication services for all use cases of 5G, namely, Enhanced Mobile Broadband (eMBB), Machine Type Communication (MTC) and Ultra-reliable Low-latency Communication (URLLC) [2]. However, high atmospheric absorption at the mm-wave frequencies requires

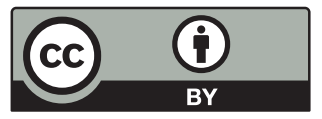

This work is licensed under a Creative Commons Attribution 4.0 International License, which permits unrestricted use, distribution, and reproduction in any medium, provided the original work is properly cited. 
antennas with high gain and increased directivity. In order to achieve these propitious characteristics promised by the $5 \mathrm{G}$ standards, suitable antennas characterized by a compact size, a large bandwidth, as well as a high gain are strongly required [3,4].

Recently, various studies are conducted to obtain antenna designs to fulfill the requirements of future wireless communication technologies. The antennas capable of end fire radiation with high gain and increased directivity are prominent among these designs [5-10]. Several designs have been reported for $5 \mathrm{G} \mathrm{mm-wave} \mathrm{applications} \mathrm{[11-21].} \mathrm{A} \mathrm{dual} \mathrm{polarized} \mathrm{end-fire} \mathrm{phased} \mathrm{array,} \mathrm{with}$ 4 elements, having $25 \%$ bandwidth at $-6 \mathrm{~dB}$ matching is presented. The reported gain is $7.48-$ $8.14 \mathrm{dBi}$ and $4.49-8.05 \mathrm{dBi}$, for horizontal and vertical polarizations, respectively [11]. Similarly, 8-element reconfigurable phased array with planar structure has reported gain of about $10 \mathrm{dBi}$ and $4.3 \mathrm{dBi}$ for single element in end-fire mode [12], whereas, 4-element reconfigurable phased array with gain in the range of 5.3-8.2 $\mathrm{dBi}$ for end-fire mode has also been presented [13]. Hong et al. reported 16-element mesh-grid patch antenna array where the single element has gain of $3.5 \mathrm{dBi}$ [14], whereas, Ta et al. has reported 8-element array with planar single element having gain of 4.5-5.8 $\mathrm{dBi}$ [15]. A MIMO antenna consisting of radiating patch with air filled slots demonstrated gain of $5.13 \mathrm{dBi}[16]$. It can be deduced from the above discussion that single element with lower gains were used to achieve higher gains [11-16]. Here the presented antenna has measured gain in the range of 4 to $6 \mathrm{~dB}$, which is suitable for $5 \mathrm{G} \mathrm{mm}$-wave applications.

In addition, various helical antennas are reported exhibiting higher gain and directivity, as well as large operational bandwidth [17-22]. However, the conventional helical antennas suffer from the main shortcoming of high profile with increased physical height. Consequently, this led the researchers to conduct studies in order to obtain a compact helical antenna with low profile, however maintaining the merits of high gain and directivity as required by the future communication systems. Therefore, planar helix antennas are realized by the researchers, especially for the mm-wave frequency range. In [19], a low-profile helical antenna is proposed, where the antenna is realized to be planar with printed strips and plated via holes to obtain wideband and end-fire radiation pattern. In [20], a wideband printed antenna based on the classical end fire-mode helical antenna with curved microstrip transmission lines is proposed. The antenna array obtains very high gain, however the design has increased fabrication complexity. In [21], planar configuration is employed to obtain a low-profile helical antenna with reduced length. The proposed antenna is composed of 6 arm patterns placed on the top and bottom side of the substrate and connected through vias. Another work in [22] presented a compact wideband end fire antenna using planar technology. The antenna is designed using two Rogers 4350 PCBs adhered together to form an RF PCB with approx. $3 \mathrm{~mm}$ thickness. This helix antenna obtains compactness, wide bandwidth, and high gain. The helical antenna designs discussed above are planar and compact in structure however multiple vias are integrated to connect the upper and bottom helical arms, which increases the level of complexity as well as the manufacturing cost of the design.

Therefore, in order to address the aforementioned challenges this paper presents a 5G broadband antenna inspired by the planar helix-shaped antenna. The major contribution of the proposed work can be summarized as follows:

- The presented work is characterized by simple geometrical structure, compact size, broad bandwidth, and high gain.

- The presented work was inspired by helix antennas without employing any vias, thus significantly reducing the design complexity of end-fire antennas. 
- Strong agreement between simulated and measured results makes the proposed work a potential candidate for $5 \mathrm{G}$ communications and for sensing, tracking, internet of things (IoT), and imaging applications using $28 \mathrm{GHz}$ frequency band.

\section{Antenna Design and Characterization}

\subsection{Geometrical Configuration}

Figs. 1a-1c depicts the top, bottom and side geometrical configuration of the proposed endfire antenna. The geometry of the proposed antenna is imprinted on $0.203 \mathrm{~mm}$ thick ROGERS RO4003 substrate having $\varepsilon \mathrm{r}=3.38$ and $\tan \delta=0.0027$. Grounded Co-Planar Waveguide (GCPW) technique is utilized to excite the antenna having partial ground plane as depicted in Fig. 1. A hole is etched from substrate and CPW ground plane for the insertion of the screw of end launch connector used for testing purpose. The end launch connector has been used to demonstrate the concept due its better performance and ease of usage at $28 \mathrm{GHz} \mathrm{mm}$-wave designs [23-27].

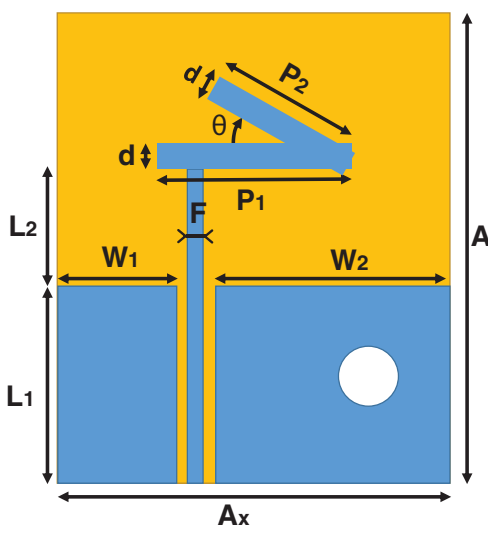

(a)

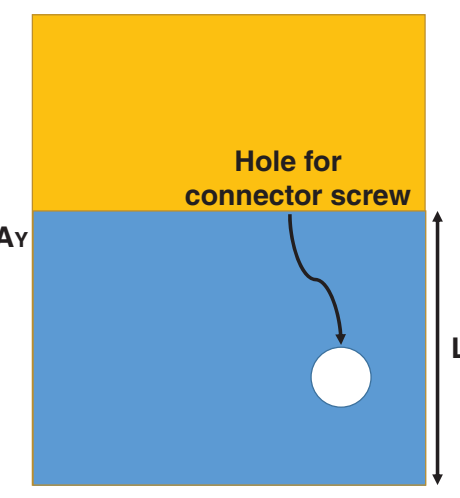

(b)

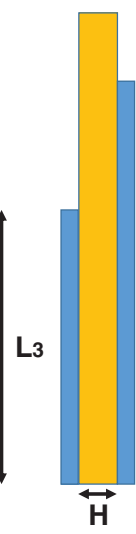

(c)

Figure 1: Geometry of proposed end-fire antenna (a) top view (b) bottom view (c) side view

\subsection{Antenna Design}

The antenna design consists of three major steps as depicted in Fig. 2a. In step-1, a T-shaped printed antenna is designed to resonate at $28 \mathrm{GHz}$, the resultant antenna shows impedance bandwidth ranging $27.8-28.4 \mathrm{GHz}$, as depicted in Fig. 2. For the desired central frequency, the length of the T-shaped antenna could be estimated by using the relations provided in [28]. Afterwards, the T-shaped antenna is modified by utilizing asymmetric CPW virtual ground. Due to the asymmetric geometrical structure, the antenna starts exhibiting an end-fire radiation pattern having a broad bandwidth of 26.9 to $31.1 \mathrm{GHz}$. Detailed numerical analysis is performed to get the wide bandwidth having maximum achievable gain.

At last, in step-3, another rectangular strip is added to one end of a modified T-shaped radiator at an angle of $\theta=30^{\circ}$. The angle between two arms of the radiator was numerically investigated and an optimized value of $\theta=30^{\circ}$ is chosen to get maximum bandwidth and gain in end-fire direction. In [26] researcher provides a detail discussion on theoretical and mathematical analysis on choosing the angle between two arms and its effect on various performance parameters. The resultant geometry is inspired by a single turn planar helical antenna without utilizing 
any vias. The resultant antenna shows a $-10 \mathrm{~dB}$ impedance bandwidth of 26.15 to $29.8 \mathrm{GHz}$, as depicted in Fig. 2a. It is worth noting that the dimension of the ground plane at the back of the substrate remains unchanged for all three steps. Tab. 1 presents the various parameters of the optimized antenna.

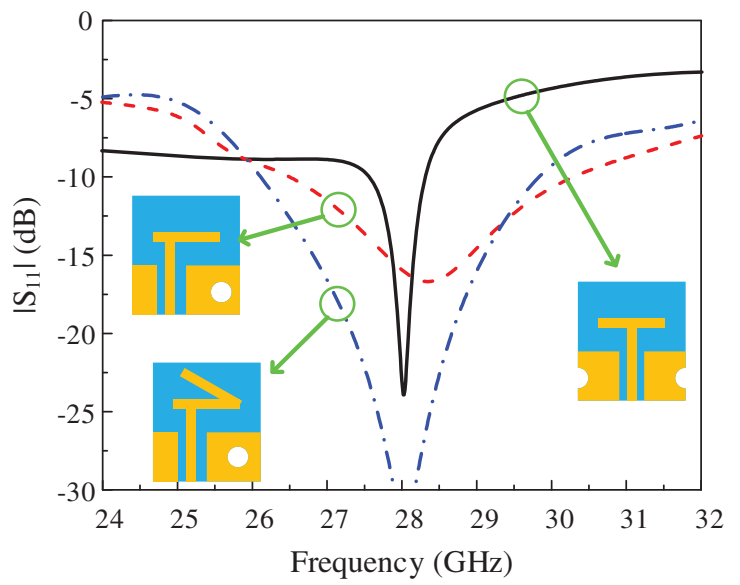

(a)

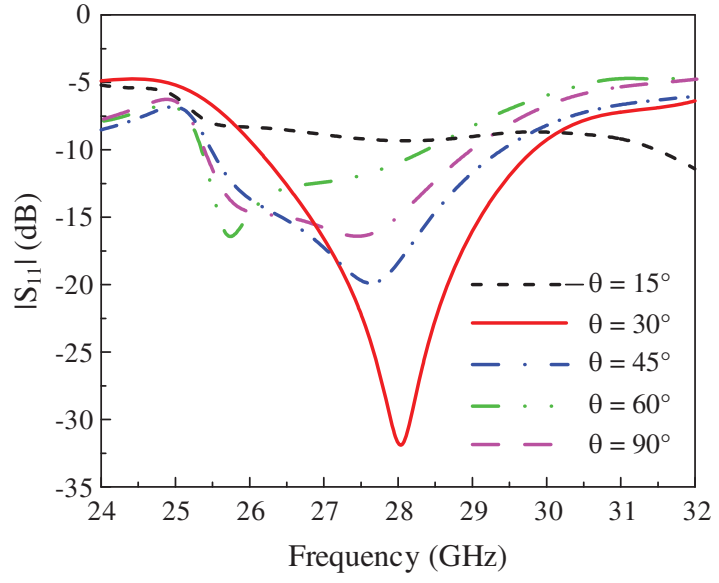

(b)

Figure 2: (a) Predicted $\left|S_{11}\right|$ comparison of various steps in evolution of the proposed antenna (b) Parametric analysis of the angle $(\theta)$ between two arms of the proposed antenna design

Table 1: The optimized parameters of the final proposed design

\begin{tabular}{llll}
\hline Parameter & Value $(\mathrm{mm})$ & Parameter & Value $(\mathrm{mm})$ \\
\hline $\mathrm{A}_{\mathrm{Y}}$ & 15 & $\mathrm{~A}_{\mathrm{X}}$ & 10 \\
$\mathrm{~L}_{1}$ & 5 & $\mathrm{~L}_{2}$ & 3 \\
$\mathrm{~L}_{3}$ & 7 & $\mathrm{H}$ & 0.203 \\
$\mathrm{~F}$ & 0.45 & $\mathrm{P}_{1}$ & 5 \\
$\mathrm{P}_{2}$ & 4 & $\theta$ & $30^{\circ}$ \\
$\mathrm{W}_{1}$ & 3 & $\mathrm{~W}_{2}$ & 6 \\
$\mathrm{D}$ & 0.7 & & \\
\hline
\end{tabular}

\subsection{Parametric Analysis}

The angle $(\theta)$ between the arms of the helix, also known as pitch angle, is one of the key parameters in designing helix antennas. A parametric analysis of the pitch angle is performed to investigate its effects on the performance of the design. It is observed from Fig. 2b, that when the pitch angle is shifted from $30^{\circ}$ to $15^{\circ}$, the resonance of the antenna is shifted to higher frequencies. Contrary to this, when the angle is changed from $30^{\circ}$ to $45^{\circ}$, the antenna shifts its resonance toward the lower side and a small decrement in bandwidth along with the impedance mismatch is also noted. Mismatch and frequency shift towards the lower side is also noted when pitch angle is further changed to $60^{\circ}$ and $90^{\circ}$. Thus, it can be concluded from the analysis that for desired applications the optimized pitch angle is $30^{\circ}$, as depicted in Fig. $2 \mathrm{~b}$. 


\section{Results and Discussion}

\subsection{Return Loss}

To validate the performance of the proposed broadband antenna a sample prototype is fabricated and tested, as shown in the inset of Fig. 3. The s-parameters for the simulated and measured case of the proposed antenna are shown in Fig. 3. The simulated $-10 \mathrm{~dB}$ impedance bandwidth is observed to be $3.65 \mathrm{GHz}$ ranging from 26.15 to $29.8 \mathrm{GHz}$ while the measured bandwidth is observed to $3.89 \mathrm{GHz}$ ranging $26.25-30.14 \mathrm{GHz}$, as depicted in Fig. 3. It is worth noting that the operational bandwidth of the antenna covers the frequency band spectrum (26.5-29.5 GHz) allocated for 5G mm-wave applications.

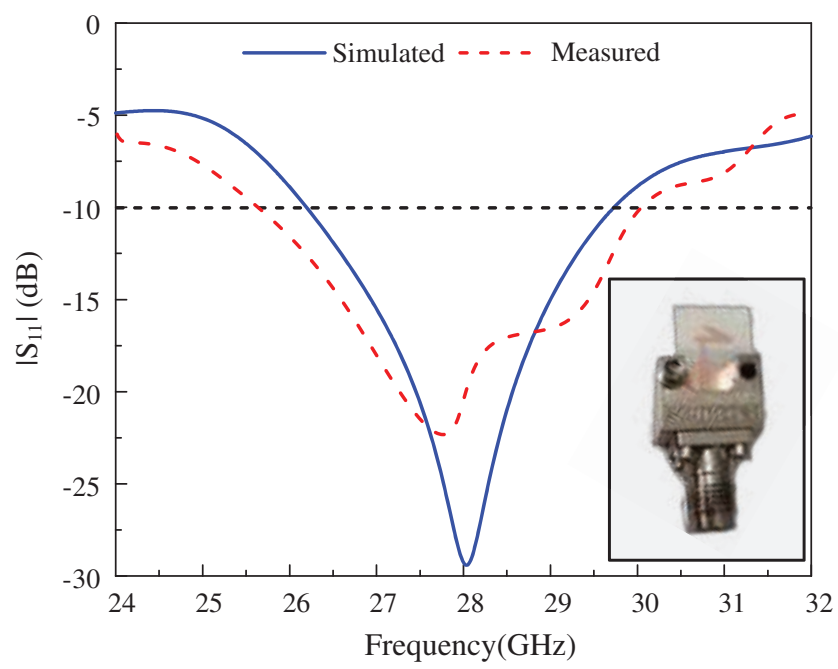

Figure 3: Comparison of simulated and measured return loss of proposed antenna

\subsection{Current Distribution}

Fig. 4 show the current distribution on the antenna. It can be noted that the stronger currents are present at the longer edge of the T-shaped radiator and extended radiator connected to it at the pitch angle. It can also be deduced from the parametric analysis presented in Fig. 2 that with the smaller pitch angle (i.e., $15^{\circ}$ ) the currents in the arms of the vertex becomes more parallel and with opposing impact. For other values, the current has less coupling between the arms of the vertex, thus strong resonance is observed.

\subsection{Far Field Analysis}

Fig. 5 presents the comparison among numerically predicted and measured radiation patterns of the proposed antenna. It can be seen from the Fig. 5 that the antenna exhibits end-fire radiation pattern at $28 \mathrm{GHz}$ with the main beam pointed toward $\theta=255^{\circ}$ in principle E-Plane $\left(\Phi=0^{\circ}\right)$ while the main beam is pointed toward $\theta=235^{\circ}$ in principle H-plane $\left(\Phi=90^{\circ}\right)$. The antenna has simulated peak gain of 5.92 dB. Fig. 6 illustrates the comparison among simulated and measured peak gain along with numerically calculated radiation efficiency. The proposed broadband antenna exhibits a peak gain of $5.83 \mathrm{~dB}$ at $28 \mathrm{GHz}$ while the radiation efficiency up to $85 \%$ is noted. 


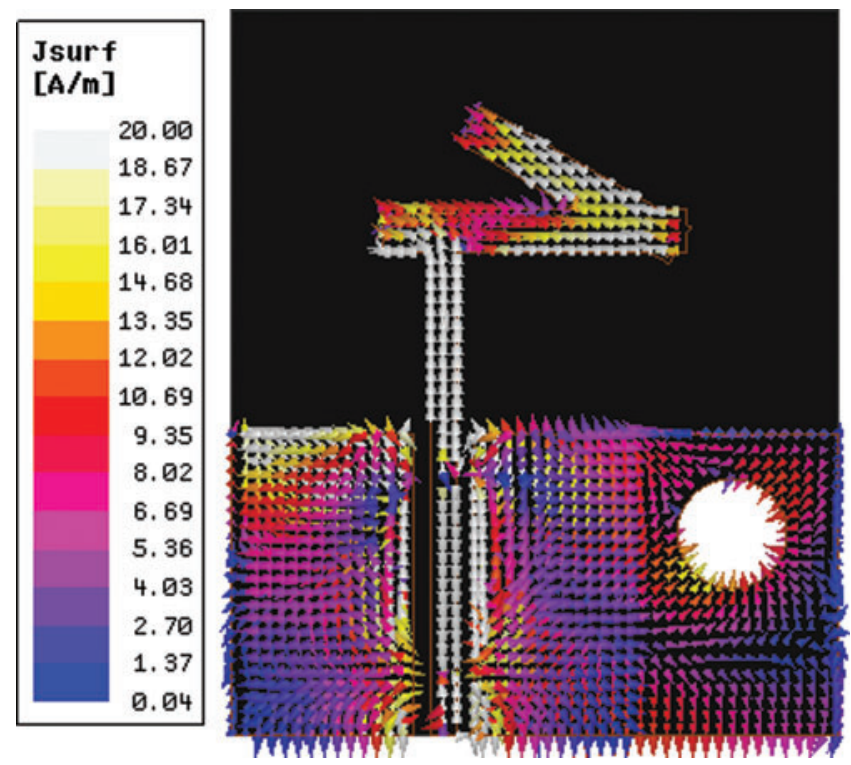

Figure 4: Current distribution of the proposed antenna at $28 \mathrm{GHz}$

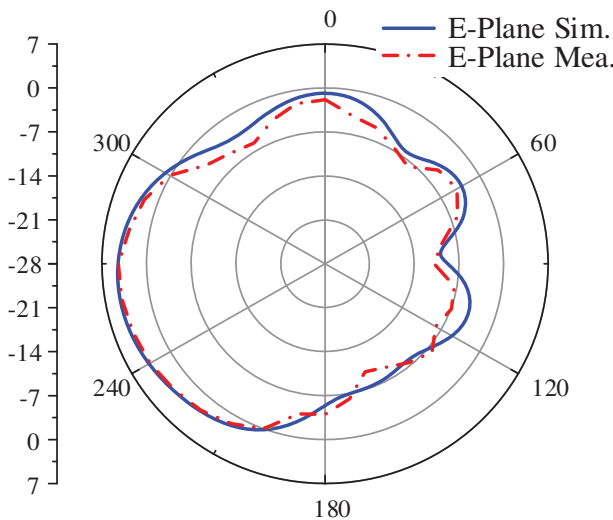

(a)

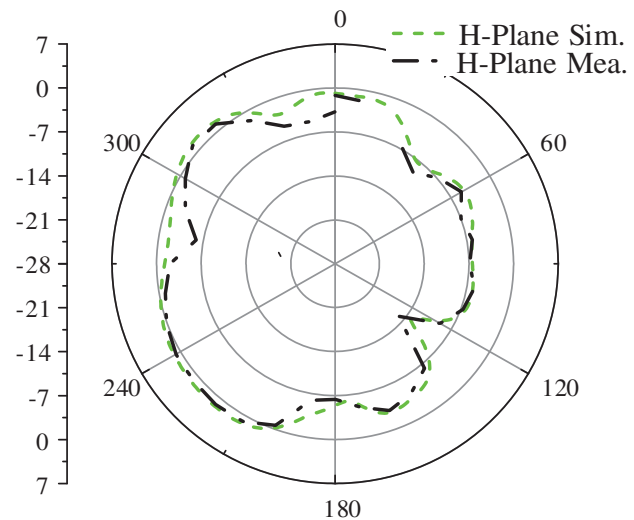

(b)

Figure 5: Comparison of simulated and measured radiation pattern at $28 \mathrm{GHz}$ (a) E-plane (b) H-plane

\subsection{Array Configuration}

The proposed design has further been analyzed for linear array configurations where higher gain will be desired. The linear array will be more suitable for applications with size constraints in one dimension. Linear array of 2 and 4 elements with in-phase feed and side-by-side arrangement as shown in Fig. 7 has been investigated. The distance (d) is half wavelength from edge of the radiating element to the edge of the other adjacent radiating element. Center to center distance (d) is not considered due to the size of the connector. By controlling the phase delay in feeding the elements, the radiation beam can be tilted. MIMO (Multiple Input Multiple Output) configurations with side-by-side and orthogonal arrangements are suitable for diversity applications [29-32]. 
As shown in Fig. 8a gain of $8.23 \mathrm{~dB}$ and $11.10 \mathrm{~dB}$ has been noted for linear array of 2 and 4 elements, respectively.

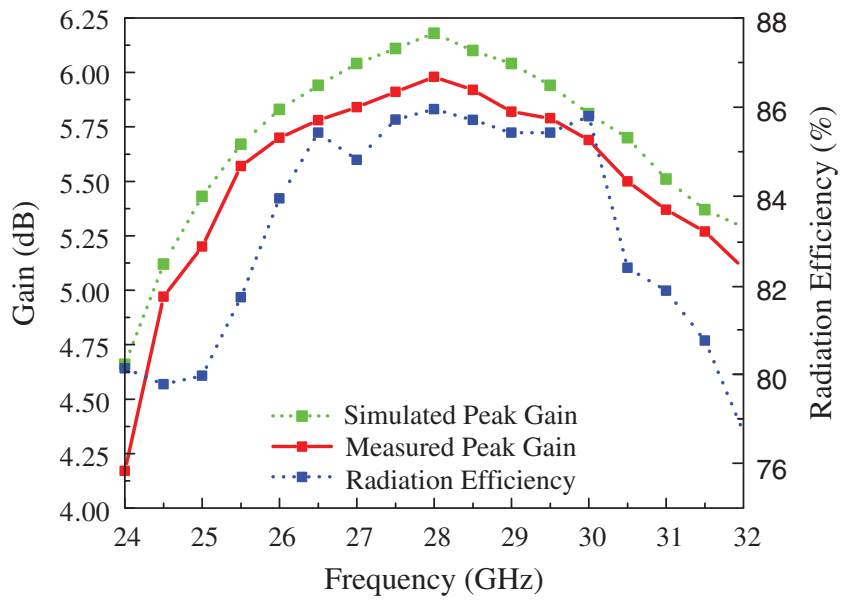

Figure 6: Comparison of simulated and measured peak gain of the proposed antenna along with its radiation efficiency

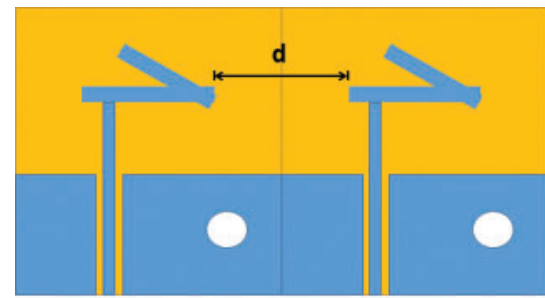

(a)

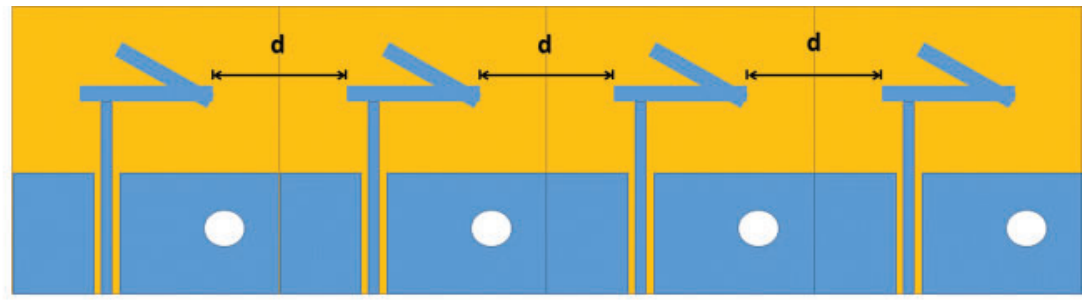

(b)

Figure 7: Array configuration for (a) 2 elements array (b) 4 elements array

\begin{tabular}{|c|}
\hline$d B$ (GainTota 1 ) \\
\\
8.23 \\
5.68 \\
3.13 \\
0.58 \\
-1.96 \\
-4.51 \\
-7.06 \\
-9.61 \\
-12.16 \\
-14.71 \\
-17.26 \\
-19.81 \\
-22.35 \\
-24.90 \\
-27.45 \\
-30.00 \\
\hline
\end{tabular}

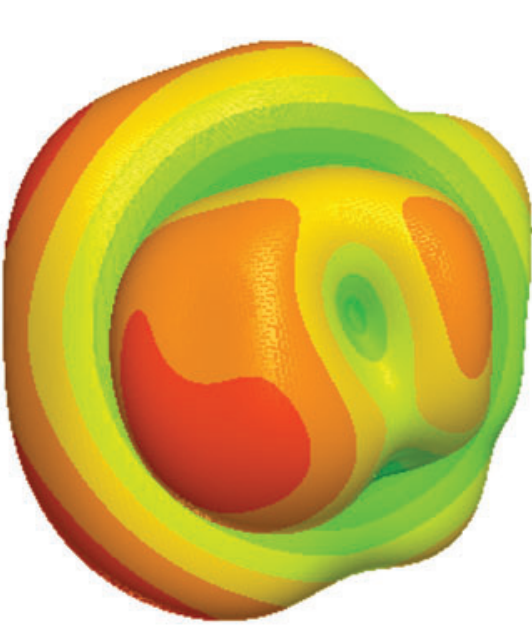

(a)
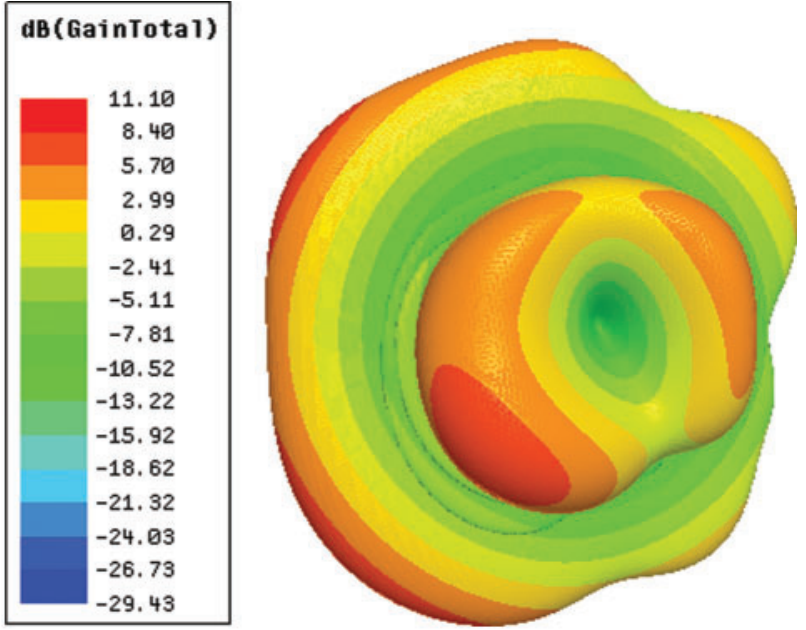

(b)

Figure 8: Predicted radiation pattern at $28 \mathrm{GHz}$ for (a) 2 elements array (b) 4 elements array 


\subsection{Comparison with State of the Art Work}

Tab. 2 summarizes the comparison of the proposed antenna with recently reported antennas exhibiting analogous features. It is evident from this comparison that the antenna proposed in this work outperforms the other similar antennas by demonstrating compact size with large fractional bandwidth and sufficient gain. In addition, the proposed design is via free, whereas the other designs incorporated vias which increases the structural complexity and thus limits the suitability of these design for future communication systems.

Table 2: Comparison of proposed work with end-fire antennas

\begin{tabular}{lllll}
\hline Ref. & Dimension $\left(\lambda_{0} \times \lambda_{0}\right)$ & Fractional bandwidth $(\%)$ & Peak gain $(\mathrm{dB})$ & Via free design \\
\hline$[5]$ & $0.5 \times 0.3$ & 3 & 3.2 & No \\
{$[6]$} & $5.6 \times 1.6$ & 11.6 & 11.8 & Yes \\
{$[7]$} & $3.1 \times 1.7$ & 11.6 & 12.5 & No \\
{$[10]$} & $3.1 \times 1.7$ & 51.8 & 12.5 & No \\
{$[19]$} & $1.27 \times 1.23$ & 54 & 10.5 & No \\
{$[21]$} & $3 \times 1.4$ & 25.5 & 6.3 & No \\
Proposed & $1.36 \times 0.9$ & 14 & 5.9 & Yes \\
\hline
\end{tabular}

\section{Conclusion}

A planar single turn helix inspired antenna having a wideband operation with an end-fire radiation pattern is presented for $5 \mathrm{G} \mathrm{mm}$-wave applications. The antenna was extracted from a T-shaped shaped monopole antenna, where T-shaped is modified to asymmetric geometry to get a directional radiation pattern along with wideband. A prototype is fabricated for measurement results. The antenna has overall compact dimensions of $1.36 \lambda_{0} \times 0.9 \lambda_{0} \times 0.0189 \lambda_{0}$. The antenna offers a wide measured impedance bandwidth ranging from $26.25-30.14 \mathrm{GHz}$ along with measured gain up to $5.83 \mathrm{~dB}$ and measured radiation efficiency up to $85 \%$. For applications with size constraints in one-dimension, linear array with 2 and 4 elements have been analyzed to demonstrate high gain.

Funding Statement: The authors received no specific funding for this study.

Conflicts of Interest: The authors declare that they have no conflicts of interest to report regarding the present study.

\section{References}

[1] J. G. Andrews, S. Buzzi, W. Choi, S. V. Hanly, A. Lozano et al., "What will 5G be?," IEEE Journal on Selected Areas in Communications, vol. 32, no. 6, pp. 1065-1082, 2014.

[2] M. Bozic, M. Cabarkapa, D. Barjamovic and D. Budimir, "Waveform comparison and PA nonlinearity effects on CP-OFDM and 5G FBMC wireless systems," Microwave and Optical Technology Letters, vol. 60, no. 8, pp. 1952-1956, 2018.

[3] N. Hussain, M. Jeong, A. Abbas, T. Kim and N. Kim, "A metasurface-based low-profile wideband circularly polarized patch antenna for 5G millimeter-wave systems," IEEE Access, vol. 8, pp. 2212722135, 2020. 
[4] S. F. Jilani, Q. H. Abbasi, Z. U. Khan, T. H. Loh and A. Alomainy, "A Ka-band antenna based on an enhanced franklin model for 5G cellular networks," Microwave and Optical Technology Letters, vol. 60, no. 6, pp. 1562-1566, 2018.

[5] J. Park, D. Choi and W. Hong, " $28 \mathrm{GHz} 5 \mathrm{G}$ dual-polarized end-fire antenna with electrically-small profile," in 12th European Conf. on Antennas and Propagation (EuCAP 2018), London, UK, pp. 1-4, 2018.

[6] A. R. Guraliuc, N. Chahat, C. Leduc, M. Zhadobov and R. Sauleau, "End-fire antenna for BAN at 60 GHz: Impact of bending, on-body performances, and study of an on to off-body scenario," Electronics, vol. 3, no. 2, pp. 221-233, 2014.

[7] A. Dadgarpour, B. Zarghooni, B. S. Virdee and T. A. Denidni, "Millimeter-wave high-gain SIW end-fire bow-tie antenna," IEEE Transactions on Antennas and Propagation, vol. 63, no. 5, pp. 2337-2342, 2015.

[8] A. H. Naqvi, J. H. Park, C. W. Baek and S. Lim, "Via-monopole based quasi yagi-uda antenna for W-band applications using through glass silicon via (TGSV) technology," IEEE Access, vol. 8, pp. 95139519, 2020.

[9] Q. Wu, J. Hirokawa, J. Yin, C. Yu, H. Wang et al., "Millimeter-wave multibeam endfire dual-circularly polarized antenna array for 5G wireless applications," IEEE Transactions on Antennas and Propagation, vol. 66, no. 9, pp. 4930-4935, 2018.

[10] V. Rafiei, S. Karamzadeh and H. Saygin, "Millimetre-wave high-gain circularly polarised SIW end-fire bow-tie antenna by utilising semi-planar helix unit cell," Electronics Letters, vol. 54, no. 7, pp. 411-412, 2018.

[11] J. Zhang, K. Zhao, L. Wang, S. Zhang and G. F. Pedersen, "Dual-polarized phased array with end-fire radiation for 5G handset applications," IEEE Transactions on Antennas and Propagation, vol. 68, no. 4, pp. 3277-3282, 2020.

[12] J. Zhang, S. Zhang, X. Lin, Y. Fan and G. Pedersen, "3D radiation pattern reconfigurable phased array for transmission angle sensing in 5G mobile communication," Sensors, vol. 18, no. 12, pp. 4204, 2018.

[13] J. Zhang, S. Zhang, Z. Ying, A. S. Morris and G. F. Pedersen, "Radiation-pattern reconfigurable phased array with p-i-n diodes controlled for 5G mobile terminals," IEEE Transactions on Microwave Theory and Techniques, vol. 68, no. 3, pp. 1103-1117, 2020.

[14] W. Hong, K. Baek, Y. Lee and Y. G. Kim, "Design and analysis of a low-profile $28 \mathrm{GHz}$ beam steering antenna solution for future 5G cellular applications," in IEEE MTT-S Int. Microwave Symp. (IMS2014), Tampa, FL, USA, pp. 1-4, 2014.

[15] S. X. Ta, H. Choo and I. Park, "Broadband printed-dipole antenna and its arrays for 5G applications," IEEE Antennas and Wireless Propagation Letters, vol. 16, pp. 2183-2186, 2017.

[16] H. M. Marzouk, M. I. Ahmed and A. A. Shaalan, "A novel dual-band 28/38 GHz AFSL MIMO antenna for 5G smartphone applications," Journal of Physics: Conference Series, vol. 1447, pp. 012025, 2020.

[17] C. Liu, Y. X. Guo and S. Xiao, "Circularly polarized helical antenna for ISM-band ingestible capsule endoscope systems," IEEE Transactions on Antennas and Propagation, vol. 62, no. 12, pp. 6027-39, 2014.

[18] Z. Ren, S. S. Qi, Z. Hu, Z. Shen and W. Wu, "Wideband water helical antenna of circular polarization," IEEE Transactions on Antennas and Propagation, vol. 67, no. 11, pp. 6770-77, 2019.

[19] Z. Z. Chen and Z. Shen, "Planar helical antenna of circular polarization," IEEE Transactions on Antennas and Propagation, vol. 63, no. 10, pp. 4315-4323, 2015.

[20] Y. Yang, B. Sun and J. Guo, "A single-layer wideband circularly polarized antenna for millimeter-wave applications," IEEE Transactions on Antennas and Propagation, vol. 68, no. 6, pp. 4925-4929, 2020.

[21] A. H. Naqvi, J. H. Park, C. W. Baek and S. Lim, "V-band end-fire radiating planar micromachined helical antenna using through-glass silicon via (TGSV) technology," IEEE Access, 7, pp. 87907-87915, 2019.

[22] Y. Mao and E. Shiju, "A compact wideband $24 \mathrm{GHz}$ end-fire helix antenna with high gain turn ratio in planar technology," Progress in Electromagnetics Research Letters, vol. 89, pp. 121-125, 2020. 
[23] R. A. Alhalabi and G. M. Rebeiz, "High-efficiency angled-dipole antennas for millimeter-wave phased array applications," IEEE Transactions on Antennas and Propagation, vol. 56, no. 10, pp. 3136-3142, 2008.

[24] K. Muzaffar, M. I. Magray, G. S. Karthikeya and S. K. Koul, "Wideband high aperture efficiency antennas with beam switching for mmWave 5G base stations," International Journal of RF and Microwave Computer-Aided Engineering, vol. 30, no. 8, pp. e22254, 2020.

[25] S. K. Koul, G. S. Karthikeya, A. K. Poddar and U. L. Rohde, "Compact antenna designs for future mmWave 5G smart phones," Microwave Journal, vol. 63, no. 11, pp. 22-40, 2020.

[26] G. S. Karthikeya, M. P. Abegaonkar and S. K. Koul, "CPW fed wideband corner bent antenna for 5G mobile terminals," IEEE Access, vol. 7, pp. 10967-10975, 2019.

[27] G. S. Karthikeya, S. K. Koul, A. K. Poddar and U. Rohde, "Ultra-compact orthogonal pattern diversity antenna module for 5G smartphones," Microwave Optical Technology Letters, vol. 63, no. 8, pp. 2003-2012, 2020.

[28] A. Zaidi, W. A. Awan, A. Baghdad, N. Hussain, A. Ballouk et al., "Compact size T-shaped patch antenna for E-band applications," in Int. Conf. on Wireless Networks and Mobile Communications (WINCOM), Fez, Morocco, pp. 1-3, 2019.

[29] H. Zahra, W. A. Awan, W. A. E. Ali, N. Hussain, S. M. Abbas et al., "A 28 GHz broadband helical inspired End-fire antenna and Its MIMO configuration for 5G pattern diversity applications," Electronics, vol. 10, no. 4, pp. 405, 2021.

[30] H. Lin and Y. Lin, "Millimeter-wave MIMO antennas with polarization and pattern diversity for 5G mobile communications: The corner design," in IEEE Int. Symp. on Antennas and Propagation \& USNCIURSI National Radio Science Meeting, San Diego, CA, USA, pp. 2577-2578, 2017.

[31] I. Elfergani, J. Rodriguez, A. Iqbal, M. Sajedin, C. Zebiri et al., "Compact millimeter-wave MIMO antenna for 5G applications," in European Conf. on Antennas and Propagation (EuCAP), Copenhagen, Denmark, pp. 1-5, 2020.

[32] M. Khalid, S. Iffat Naqvi, N. Hussain, M. Rahman, Fawad et al., "4-Port MIMO antenna with defected ground structure for 5G millimeter wave applications," Electronics, vol. 9, no. 1, pp. 71, 2020. 